\section{BREAST CANCER FAST, POSITIVE DATA FROM NEOSPHERE}

Before the advent of HER2-targeted therapies, the $20-25 \%$ of patients with breast tumors that overexpressed HER2 had a poor prognosis. The approval and use of the HER2-targeted monoclonal antibody trastuzumab has gone some way to ameliorating this problem; however, more treatment options are still needed.

One experimental therapy that might act in parallel with trastuzumab is another HER2-targeted monoclonal antibody, pertuzumab. This antibody targets a different area on the HER2 receptor to trastuzumab and so the rationale was that the two therapies would act in concert. A recent study (NeoSphere) has assessed the combination in the neoadjuvant setting in 417 treatment-naive patients with locally advanced, inflammatory or early stage HER2-positive breast cancer.

The patients were randomly assigned to one of four regimens: group $A$ received trastuzumab and docetaxel; group B received pertuzumab, trastuzumab and docetaxel; group $C$ received pertuzumab and trastuzumab; and group $D$ received pertuzumab and docetaxel. The primary end point of the trial was pathological complete response in the breast, which was assessed at surgery after receiving four 3-weekly cycles of therapy. Importantly, this is a short time period over which to assess a clinically meaningful primary end point, a considerable advantage for carrying out clinical trials in this setting.

Patients in group B had a significantly improved pathological complete response rate than those in group $\mathrm{A}$ who were receiving the current therapy (45.8\% versus $29.0 \%$ ). Regimens $A$ and $B$ both achieved better results than the other two experimental arms (16.8\% and $24.0 \%$ for arms $\mathrm{C}$ and $\mathrm{D}$, respectively). The authors state that "importantly, the combination of pertuzumab with trastuzumab did not result in any additional safety signals" and that, therefore, "this triplet combination [trastuzumab, pertuzumab and docetaxel] could be an attractive proposition for patients with HER2-positive breast cancer."

These data are being followed up in an ongoing phase III trial in the adjuvant setting (APHINITY), the results from this trial will be awaited with interest.

Rebecca Kirk

Original article Gianni, L. et al. Efficacy and safety of neoadjuvant pertuzumab and trastuzumab in women with locally advanced, inflammatory, or early HER2-positive breast cancer (NeoSphere): a randomised multicentre, open-label, phase 2 trial. Lancet Oncol. doi:10.1016/ S1470-2045(11)70336-9 